\title{
Author's Reply to: “Comment on: Pharmacokinetics and Safety of Recombinant Human Interleukin-1 Receptor Antagonist GR007 in Chinese Healthy Subjects"
}

\author{
Ran Xie ${ }^{1}$. Yang Zhang ${ }^{2,5} \cdot$ Nan Zhao $^{1} \cdot$ Shuang Zhou ${ }^{1} \cdot$ Xin Wang $^{3} \cdot$ Wei Han ${ }^{4,5} \cdot$ Yan Yu $^{2} \cdot$ Xia Zhao $^{1} \cdot$ Yimin Cui $^{1}$
}

Published online: 9 September 2019

(c) The Author(s) 2019

We would like to thank Dr. Srinivas for his interest in our previous publication and his pertinent comments [1].

First, we would like to describe the preparation and administration of GR007 in detail, which was not shown in our article. This process was very simple. The freeze-dried powder was diluted with isotonic saline to prepare GR007 intramuscular (IM) injection solutions of 1,3 , and $5 \mathrm{~mL}$ for doses of 30, 90, and $150 \mathrm{mg}$, respectively. The IM injection solutions were administered as a single injection within $3 \mathrm{~min}$. We chose the gluteus maximus as the injection site because this region is free of major nerves and blood vessels. According to the volume of the IM injection, 2, 5, and $5 \mathrm{~mL}$ syringes were used for doses of 30,90 and $150 \mathrm{mg}$, respectively. All the above information has been mentioned in our clinical trial protocol.

Dr. Srinivas's argument seems to be that for IM injections, the $5 \mathrm{~mL}$ volume of solution used in our study was

Ran Xie and Yang Zhang contributed equally to this work.

This reply refers to the article available at https://doi.org/10.1007/ s13318-019-00566-8.

\section{Xia Zhao \\ zxyjk@126.com \\ $\triangle$ Yimin Cui \\ cuiymzy@126.com}

1 Department of Pharmacy, Peking University First Hospital, Beijing 100034, People's Republic of China

2 Shanghai Municipality Key Laboratory of Veterinary Biotechnology, School of Agriculture and Biology, Shanghai Jiao Tong University, Shanghai, People's Republic of China

3 Department of General Surgery, Peking University First Hospital, Beijing 100034, People's Republic of China

4 Laboratory of Regeneromics, School of Pharmacy, Shanghai Jiao Tong University, Shanghai, People's Republic of China

5 General Regeneratives (Shanghai) Limited, Shanghai, People's Republic of China infeasible. However, this issue had already been considered in the development of our protocol. Considering the characteristics of the Phase I study, one of our objectives was to assess the tolerability of the investigational drug on the premise of guaranteeing the safety of the subjects. When searching the literature, a number of articles have indicated that a volume of $5 \mathrm{~mL}$ is feasible for an IM injection to the gluteus maximus [2-4]. Moreover, this method of administering GR007 can minimize administration frequency and increase the compliance of the subjects. Furthermore, no sclerosis was found at the injection site during the administration of GR007 and no injection site response was observed in this study. Therefore, it is feasible to adopt this injection method. However, as we mentioned in our discussion section, the large volume of the injection may be a possible influencing factor that led to a disproportionate increase in the maximum concentration $\left(C_{\max }\right)$.

We reported that for further studies, administration of GR007 would be once a day for multiple consecutive days; however, this may have caused Dr. Srinivas to misunderstand that this injection needs to be administered several times a day. We are extremely sorry for causing any misunderstanding regarding this issue.

It is well known that area under the concentration vs time curve (AUC) and $C_{\max }$ are common parameters used for the assessment of dose proportionality. Moreover, the statistical methods mentioned in our article are consistent with those released by the Center for Drug Evaluation (CDE) in China [5]. Dr. Srinivas noted in his comment that for the case of dose proportionality of exposure with extended absorption and distribution stages, it would be somewhat misleading to judge it with the parameter of $C_{\max }$ [1]. He also proposed that the AUC in the plateau phase of the absorption/distribution phase divided by the time duration might be a better parameter to examine it [1]. However, when retrieving the literature, we did not find any relevant articles using this parameter. Moreover, how to define the $C$ initial time point 
and ending time point lacks a unified standard. Therefore, this parameter lacks general applicability. Whether this parameter can be used in this case remains to be unverified. Therefore, we do not think that it is inappropriate to use $C_{\max }$ as a parameter for the assessment of dose proportionality.

Dr. Srinivas mentioned in his comment that keeping the same injection volume of GR007 in different dose groups is a better way to reduce the bias caused by different injection volumes [1]. We agree with him, but as this approach may increase the cost and prolong the development timeline, it may be somewhat difficult for pharmaceutical enterprises to conduct.

\section{Compliance with Ethical Standards}

Funding No external sources of funding were used in the preparation of this manuscript.

Conflicts of interest Yang Zhang and Wei Han are part-time employees of General Regeneratives (Shanghai) Limited which sponsored the clinical trial. Other authors have no relevant conflicts of interest.

Open Access This article is distributed under the terms of the Creative Commons Attribution-NonCommercial 4.0 International License (http://creativecommons.org/licenses/by-nc/4.0/), which permits any noncommercial use, distribution, and reproduction in any medium, provided you give appropriate credit to the original author(s) and the source, provide a link to the Creative Commons license, and indicate if changes were made.

\section{References}

1. Srinivas NR. Comment on: Pharmacokinetics and safety of recombinant human Interleukin-1 receptor antagonist GR007 in healthy Chinese subjects. Eur J Drug Metab Pharmacokinet. 2019. https ://doi.org/10.1007/s13318-019-00566-8.

2. Hopkins U, Arias CY. Large-volume IM injections: a review of best practices. Oncol Nurse Advis. 2013;4(1):32-7.

3. Wynaden D, Landsborough I, Chapman R, et al. Establishing best practice guidelines for administration of intra muscular injections in the adult: a systematic review of the literature. Contemp Nurse. 2005;20(2):267-77.

4. Leppik IE, Goel V, Rarick J, et al. Intramuscular and intravenous levetiracetam in humans: safety and pharmacokinetics. Epilepsy Res. 2010;91(2-3):289-92.

5. Ming Z, Jin Y. Confidence interval method for linear pharmacokinetic evaluation. In: Clinical safety and efficacy evaluation. Center for Drug Evaluation. 2013. http://www.cde.org.cn/dzkw.do?metho $\mathrm{d}=$ largePage\&id=312915. Accessed 09 Jan 2013. 